Article

\title{
Impact of Genetic Background on the Leaf-Protective Enzyme Activity and Hormone Levels of Maize
}

\author{
Chunxiao Liu ${ }^{1,2}$, Zongxin Li ${ }^{1,2}$, Tieshan Liu ${ }^{1,2}$, Hui Zhang ${ }^{1,2}$, Haijun Zhao ${ }^{2}$, \\ Qingcheng Wang ${ }^{1,2}$, Yonghui Shi ${ }^{3}$, Hongcui Dai ${ }^{4}$ and Kaichang Liu ${ }^{2,4, *}$ \\ 1 Maize Research Institute, Shandong Academy of Agricultural Sciences, Gong-ye North Road 202, \\ Jinan 250100, China; clickchx@163.com (C.L.); sdaucliff@sina.com (Z.L.); gongsi@vip.188.com (T.L.); \\ Zhang-hui160@163.com (H.Z.); qcwang@saas.ac.cn (Q.W.) \\ 2 National Engineering Laboratory for Wheat and Maize, Gong-ye North Road 202, Jinan 250100, China; \\ 83179266@163.com \\ 3 Jinan Soil and Fertilizer Station, Jian-she Road 76, Jinan 250004, China; shiyonghui77@163.com \\ 4 Crop Research Institute, Shandong Academy of Agricultural Sciences, Gong-ye North Road 202, \\ Jinan 250100, China; daihongcui2013@163.com \\ * Correspondence: liukc@saas.ac.cn; Tel.: +86-0531-6665-9091
}

Received: 7 September 2018; Accepted: 20 October 2018; Published: 22 October 2018

\begin{abstract}
The delay of leaf senescence, also known as the stay-green (SG) phenotype, is a trait closely associated with yield gain and resistance to many biotic/abiotic stresses. In order to increase the stress tolerance in maize, eight genetic background types were compared against the hallmarks of delayed senescence. The plant redox status and hormonal levels were tested among widespread SG and non-SG (NSG) maize lines in northern China. We found that SG maize lines showed greater activity of the reactive oxygen species (ROS) scavenging apparatus including leaf superoxide dismutases (SOD), peroxidases (POD), and catalases (CAT). Moreover, the concentration of malonaldehyde (MDA), a membrane lipid peroxidation marker, was significantly lower in SG than in NSG plants. For the hormone content, SG maize lines showed higher zeatin (ZR) and lower abscisic acid (ABA) after silking, resulting in a higher $\mathrm{ZR} / \mathrm{ABA}$ ratio. Understanding the correlation between the stay-green trait and the corresponding hallmarks of delayed senescence is an important step in promoting SG to increase yield and stress tolerance. Our findings provide valuable insight into how to promote the SG trait in specific maize lines to increase yield and stress tolerance.
\end{abstract}

Keywords: stay-green; senescence-related enzymes; endogenous hormone; maize

\section{Introduction}

The stay-green (SG) trait refers to a lack of obvious loss of green in leaves due to the delay of senescence during the physiological maturation of grain. Right after the successful pollination and initiation of kernel development, the grain fill period begins and lasts for two months until the kernels are physiologically mature. During the grain fill period, the developing kernels demand nutrition from the rest of the plant, especially from the green leaves that are undergoing active photosynthetic process. The anthesis maturity stage is the key period for determining not only maize yield, but also leaf senescence with physiological functions [1].

The early onset of leaf senescence affects assimilation and grain filling in crop plants and thus reduces crop yield [2]. In contrast, delayed leaf senescence, also called the stay-green (SG) trait, extends the active photosynthesis state and leads to a greater accumulation of dry matter in seeds, leading to a higher crop yield [3,4]. Moreover, the SG trait is also associated with an increased resistance to abiotic stresses such as heat and drought [5], and biotic stresses [6]. Researchers have found a 
positive correlation of the SG trait with a higher grain yield in crop plants such as sorghum $[7,8]$, wheat [9], and maize [10,11].

Leaf senescence is genetically controlled and is also regulated by environmental factors and developmental factors [12]. Previous research has found that abiotic stresses such as drought [13], salinity [14], nutrient deficiency [15], or extreme temperatures cause the production of reactive oxygen species (ROS) $[6,16]$. Consequently, ROS scavenging by enzymes could effectively eliminate the reactive oxygen species, slow down senescence, and enhance resistance to oxidative stress. Superoxide dismutase (SOD), peroxidase (POD), and catalase (CAT) activity are therefore considered indicators of ongoing senescence.

In addition to being a consequence of environmental stresses, senescence is also regulated by endogenous factors such as hormones. Plant hormones function as internal signals, triggering senescence and mediating processes such as the degradation of chloroplasts and photosynthetic proteins [17]. For example, abscisic acid (ABA) promotes leaf yellowing and senescence, while cytokinins (CTKs) have the opposite effect and increase the stay-green time [18,19].

Indeed, the extension of leaf senescence is a key developmental process that correlates directly to the maize grain yield [1]. Therefore, selecting maize varieties with the SG trait, or using strategies to postpone the onset of senescence and keep leaves green to allow an extended photosynthetic state, are expected to significantly increase the yield, especially during grain fill stages, or when maize plants are challenged by abiotic and biotic stresses [20].

The SG maize varieties are characterized by a higher SG ratio (proportion of green tissue on the total plant area) and no chlorosis on the green leaves, while the SG ratio of the hybrid lines follows the order SG $\times$ SG $>$ SG $\times$ NSG (non-SG) $>$ NSG $\times$ SG $>$ NSG $\times$ NSG. Understanding the hallmarks of the stay-green traits and corresponding events in SG maize at the post-silking and grain fill stages is of agronomic importance [21]. Wang et al. (1999) [22] studied major maize varieties in China and found a rapid decrease in the activity of SOD, CAT, and POD in leaves after plant anthesis, and a quick accumulation of malonaldehyde (MDA), the product of membrane lipid peroxidation. Meanwhile, in slow-decaying maize varieties, the activity of SOD, CAT, and POD in the leaves decreases more slowly and MDA accumulates at a slower rate. The difference becomes more apparent in the late silking stages.

Previous research on maize leaf senescence emphasized active oxygen metabolism under stress conditions such as nutrient deficiency, drought, extreme temperature, and salinity stresses [13,15]. The SG lines Q319 and CZ01, and the non-SG (NSG) lines BM and B73 are widespread in Northern China. Understanding the correlation of the corresponding physiological hallmarks with the SG trait is an important step in increasing the yield and stress resistance in these specific maize varieties. To achieve this, we measured the ROS scavenging activity and endogenous hormones in leaves of different senescing SG varieties at different stages after silking in their parental lines and their F1 generations between SG lines and non-SG lines. Our findings provide valuable insight into how to promote the SG trait in these specific maize lines in order to increase the yield and stress tolerance. While crop yield is a complex trait in maize, introducing the SG trait into maize lines with combinational favorable traits is a good strategy for maize breeding.

\section{Materials and Methods}

\subsection{Experimental Conditions}

The field experiment was carried out at the Liuyi Farm at the Maize Institute, Shandong Academy of Agricultural Sciences $\left(117^{\circ} 1^{\prime} \mathrm{E}, 37^{\circ} 1^{\prime} \mathrm{N}\right)$. The region has a temperate monsoon climate with an annual average precipitation of $547.5 \mathrm{~mm}$, mainly occurring from June to August and has a mean annual temperature of $12.9^{\circ} \mathrm{C}$. The soil in the field is a fertile sand-loam type with $1.11 \%$ organic matter, $0.11 \%$ total nitrogen, $53.16 \mathrm{mg} \mathrm{kg}^{-1}$ available nitrogen, and $29.38 \mathrm{mg} \mathrm{kg}^{-1}$ available phosphorus, with $87.17 \mathrm{mg} \mathrm{kg}^{-1}$ available phosphorus in the surface soil. 


\subsection{Experimental Designs}

To fully demonstrate the correlations of the corresponding physiological hallmarks with the SG trait, F1 plants of crosses [Q319 × CZ01 (SG × SG), Q319 × BM (SG $\times$ NSG), BM $\times$ Q319 $(\mathrm{NSG} \times \mathrm{SG})$, and B73 $\times$ BM $($ NSG $\times$ NSG)], and their parental lines were used for our experiment. Each cross was performed between 12 pairs of maize plants and their F1 generations and was analyzed for the hallmarks related to the SG trait (Table 1). Two SG inbred lines, Q319 [23] and CZ01 [24], and two NSG inbred lines, BM [21] and B73 [24], were used in this experiment.

Table 1. The parental and F1 materials used in this study.

\begin{tabular}{|c|c|c|}
\hline Generation & Material Code & Hybrid Lines \\
\hline \multirow{4}{*}{ Self-Cross } & SG1 & CZ01 \\
\hline & SG2 & Q319 \\
\hline & NSG1 & $\mathrm{BM}$ \\
\hline & NSG2 & B73 \\
\hline \multirow{8}{*}{ Hybrid Cross } & $\mathrm{SG} 1 \times \mathrm{SG} 2$ & $\mathrm{CZ01} \times \mathrm{Q} 319$ \\
\hline & $\mathrm{SG} 2 \times \mathrm{SG} 1$ & Q319 × CZ01 \\
\hline & $\mathrm{SG} 1 \times \mathrm{NSG} 1$ & $\mathrm{CZ} 01 \times \mathrm{BM}$ \\
\hline & $\mathrm{SG} 2 \times \mathrm{NSG} 1$ & $\mathrm{Q} 319 \times \mathrm{BM}$ \\
\hline & NSG1 $\times$ SG1 & $\widehat{\mathrm{BM}} \times \mathrm{ZC} 01$ \\
\hline & NSG1 × SG2 & $\mathrm{BM} \times \mathrm{Q} 319$ \\
\hline & $\mathrm{NSG} 1 \times \mathrm{NSG} 2$ & $\mathrm{BM} \times \mathrm{B} 73$ \\
\hline & NSG2 $\times$ NSG1 & $\mathrm{B} 73 \times \mathrm{BM}$ \\
\hline
\end{tabular}

Note: SG1 and SG2 are two types of maize for a higher stay-green ratio; CZ01, Q319, BM, B73 are the names of maize varieties.

The SG and NSG lines were planted with three replications in $19.8 \mathrm{~m}^{2}(3.96 \mathrm{~m} \times 5.0 \mathrm{~m})$ plots, in six rows, with $66 \mathrm{~cm}$ row spaces, and a density of 60,000 plants ha ${ }^{-1}$. Maize was sown on 8 June and harvested at the end of September in the same year, following conventional management practices such as irrigation and fertilization.

\subsection{Sampling and Measurements}

Three maize plants of equal size from each $\mathrm{F} 1$ generation were selected from the silking, filling, milky, and maturity stages $(0,15,30$, and 55 days after silking). Ear leaves were sampled, frozen in liquid nitrogen, and stored at $-40{ }^{\circ} \mathrm{C}$ for further experiments.

\subsection{Activity of SOD, CAT and POD, and the MDA Content}

One gram of frozen leaf fragments was ground into a homogenate in a pre-chilled mortar in an ice bath with $5 \mathrm{~mL}$ of $0.1 \mathrm{~mol} / \mathrm{L}$ PBS (phosphate buffered saline, $\mathrm{pH} 7.0$ ) and a small amount of silica sand; $5 \mathrm{~mL}$ PBS was added again during grinding. The supernatant was centrifuged at 10,000 rpm for 15 min at $4{ }^{\circ} \mathrm{C}$, transferred to pre-chilled tubes, and saved as a crude enzyme extract at $4{ }^{\circ} \mathrm{C}$ for up to $48 \mathrm{~h}$. A UV-2800 ultraviolet and visible spectrophotometer (Shimadzu, Kyoto, Japan) was used in all of the following tests.

SOD activity was determined by the reduction of nitrogen blue tetrazolium (NBT) [25] under 4000 LX fluorescent light for $15 \mathrm{~min}$; then the absorbance at $560 \mathrm{~nm}$ was measured and the enzyme activity was calculated. An enzyme unit was defined as the amount of enzyme required to reduce NBT to half the concentration of the control (50\%). The specific activity was expressed in units of enzyme per $\mathrm{mg}$ of protein enzyme (unit/mg).

POD activity was determined by the reduction of guaiacol [26]. The enzyme activity was defined as $\triangle \mathrm{OD} 470 \mathrm{~g}^{-1} \mathrm{FW} \mathrm{min}^{-1}$. CAT activity was determined by potassium permanganate titration-the amount of $\mathrm{H}_{2} \mathrm{O}_{2}$ ( $\left.\mu \mathrm{mol}\right)$ decomposed by $1 \mathrm{~g}$ of fresh weight in $1 \mathrm{~min}$ at $20^{\circ} \mathrm{C}$. The MDA content was determined by the thiobarbituric acid colorimetry method [27]. 


\subsection{Content of Endogenous Hormones}

Zeatin (ZR) and ABA were measured using an enzyme-linked immunosorbent assay (ELISA) [28]. Fresh leaf samples of 2-4 g were cut into pieces, mixed with $10 \mathrm{~mL}$ of $80 \%$ ice-methanol and ground. The sample was transferred into a $150 \mathrm{~mL}$ conical flask with a stopper. After adding ice-methanol, the mixture was sonicated for $2 \mathrm{~h}$, then kept on ice. The filtered residue was then added to $20 \mathrm{~mL}$ of $80 \%$ ice-methanol, mixed evenly, refrigerated for $12 \mathrm{~h}$ and then filtered. The filtrate was dried at $40{ }^{\circ} \mathrm{C}$, transferred into a tube containing phosphate buffer $(\mathrm{pH}$ 8.0) and chloroform, and then extracted by chloroform three times. The $\mathrm{pH}$ was adjusted to $2.5-2.8$ by formic acid, and the filtrate was extracted by chloroform three times, dried by evaporation, washed with phosphate buffer ( $\mathrm{pH} 8.0$ ), and passed through a Seppar C18 pre-column. The pre-column was washed with $2 \mathrm{~mL}$ methanol. The washing solution was collected and filtered with a filter membrane $(0.45 \mu \mathrm{m})$. The filtrate was analyzed using high-pressure liquid chromatography (Waters510, Waters company, Eschborn, Germany), with an ODSC18 column $(250 \mathrm{~mm} \times 4.6 \mathrm{~mm})$. The mobile phase was mixed with acetonitrile:methyl alcohol:acetic acid (1\%) (5:50:45) at a flow rate of $0.8 \mathrm{~mL} \mathrm{~min}^{-1}$. The detection wavelength was $254 \mathrm{~nm}$, the sensitivity was $0.5 \mathrm{AuFs}$, and an external standard was employed for quantification.

\subsection{Statistical Analysis}

The results were initially collated with Excel 2010 (Microsoft, Albuquerque, NM, USA). One-way ANOVA analysis was based on SAS 8.1 (SAS Institute, Cary, NC, USA). Charts were made using Sigmaplot 12.0 (Systat Software Inc., San Jose, CA, USA).

\section{Results}

\subsection{Superoxide Dismutase (SOD) Activity after Silking}

The SOD activity in the SG and NSG lines showed a similar trend with the highest activity occurring from the silking period to the filling period. The activity decline after the filling period indicates a drop in ROS scavenging. However, the SOD activity was significantly higher in the SG parental lines than the NSG parental lines, and the difference became larger as kernel development advanced. The SOD activity dropped by $6.92 \%$ and $7.78 \%$ from silking to filling in the SG parental lines, compared to $49.60 \%$ and $58.68 \%$ in the NSG parental lines.

The F1 plants from the SG crosses also showed greater SOD activity than the F1 plants from the NSG crosses (Figure 1). From the silking to the filling period, SOD activity dropped by $20.2 \%$ in the SG $\times$ SG lines and $44.06 \%$ in the NSG $\times$ NSG lines, while in the SG $\times$ NSG and NSG $\times$ SG lines, it dropped intermediately between the SG $\times$ SG lines and SG $\times$ NSG lines. Leaf SOD activity in the SG maize lines dropped more slowly, maintaining a relatively higher level; therefore, leaf senescence in the SG maize lines was slower due to the greater SOD scavenging of ROS, and less accumulation of toxic matter.

\subsection{Peroxidase (POD) Activity after Silking}

The POD activity in the SG and NSG lines showed a similar trend, with the greatest activity occurring from the silking to the filling period (Figure 2). From the filling to the maturity period, leaf POD activity dropped by $24.10 \%$ and $26.67 \%$ in the SG parental lines, compared to $54.85 \%$ and $57.55 \%$ in the NSG parental lines. The F1 plants from the SG crosses also showed higher POD activity than the F1 plants from the NSG crosses. From the silking to the filling period, the POD activity dropped by $17.13 \%$ in the SG $\times$ SG lines, $46.27 \%$ in the NSG $\times$ NSG lines, while it was intermediate in the SG $\times$ NSG and NSG $\times$ SG lines. In every developing stage, the SG parental lines and F1 plants of SG $\times$ SG showed greater POD activity than their NSG counterparts. Even at the later stages, the SG lines still retained high POD activity, suggesting a stronger ability to remove ROS. 

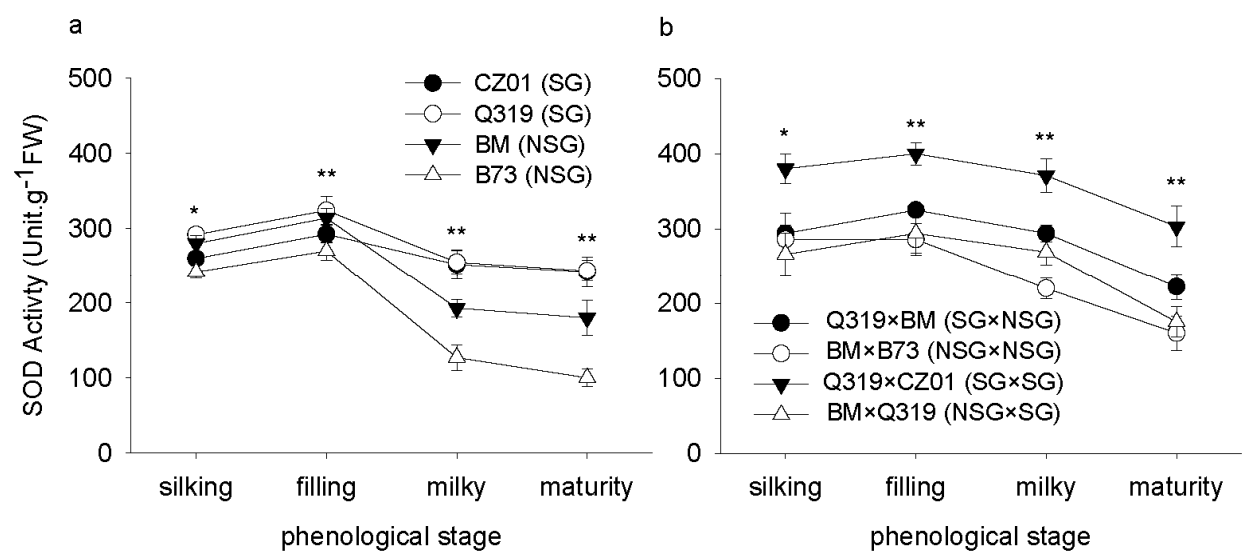

Figure 1. Superoxide dismutase (SOD) activity in ear leaves from different maize genetic backgrounds after silking. Values are means \pm standard deviation $(n=3)$. * indicates a significant difference between treatments at $p<0.05 .{ }^{* *}$ indicates a significant difference between treatments at $p<0.01$. (a) represents inbred lines, (b) represents hybrids. SG1 and SG2 are two types of maize for a higher stay-green ratio; CZ01, Q319, BM, B73 are the names of maize varieties.
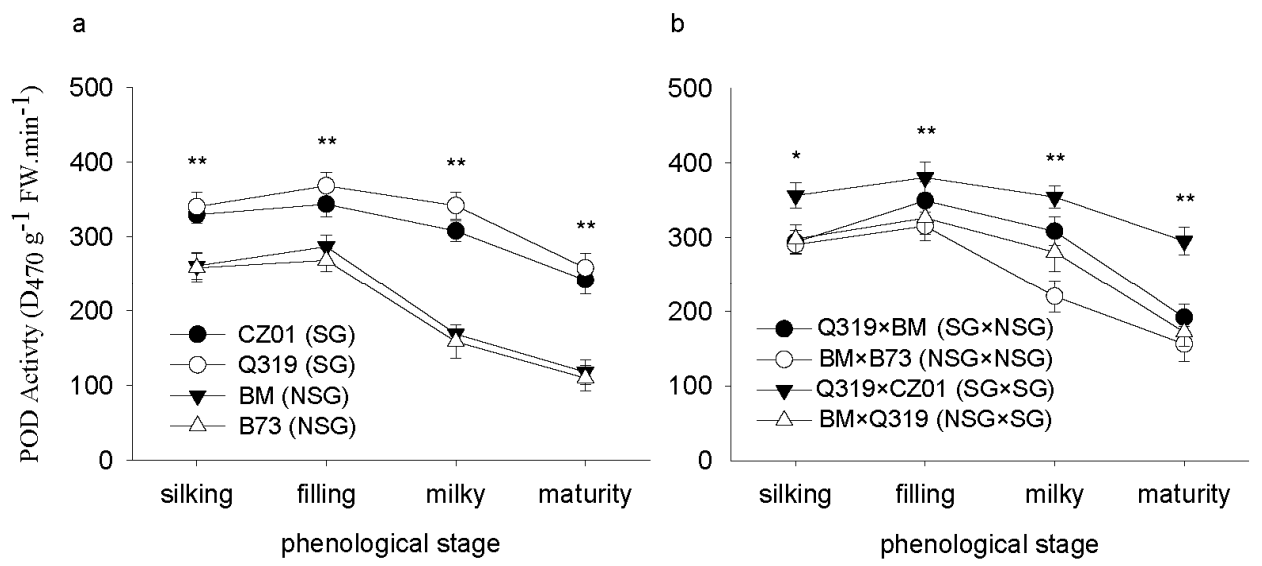

Figure 2. Peroxidase (POD) activity in ear leaves from different maize genetic backgrounds after silking. Values are means \pm standard deviation $(n=3)$. * indicates a significant difference between treatments at $p<0.05 .{ }^{* *}$ indicates a significant difference between treatments at $p<0.01$. The (a) represents inbred lines, (b) represents hybrids. SG1 and SG2 are two types of maize for a higher stay-green ratio; CZ01, Q319, BM, B73 are the names of maize varieties.

\subsection{Catalase (CAT) Activity after Silking}

The CAT activity in the leaves of all crosses was the highest from the silking to the filling stage before decreasing slowly in the SG $\times$ SG plants and more steeply in other crosses (Figure 3). CAT activity dropped by $28.42 \%$ and $30.20 \%$ from the silking to the maturity period in the SG parental lines, compared to $63.00 \%$ and $63.11 \%$ in the NSG parental lines. Activity in the SG $\times$ SG plants was higher at each growth stage when compared to other crosses, while NSG $\times$ NSG presented the lowest value. From the silking to the maturity period, activity dropped by $26.95 \%$ in the SG $\times$ SG lines, $57.21 \%$ in the NSG $\times$ NSG lines, $30.35 \%$ in the SG $\times$ NSG, and $34.58 \%$ in the NSG $\times$ SG lines. 

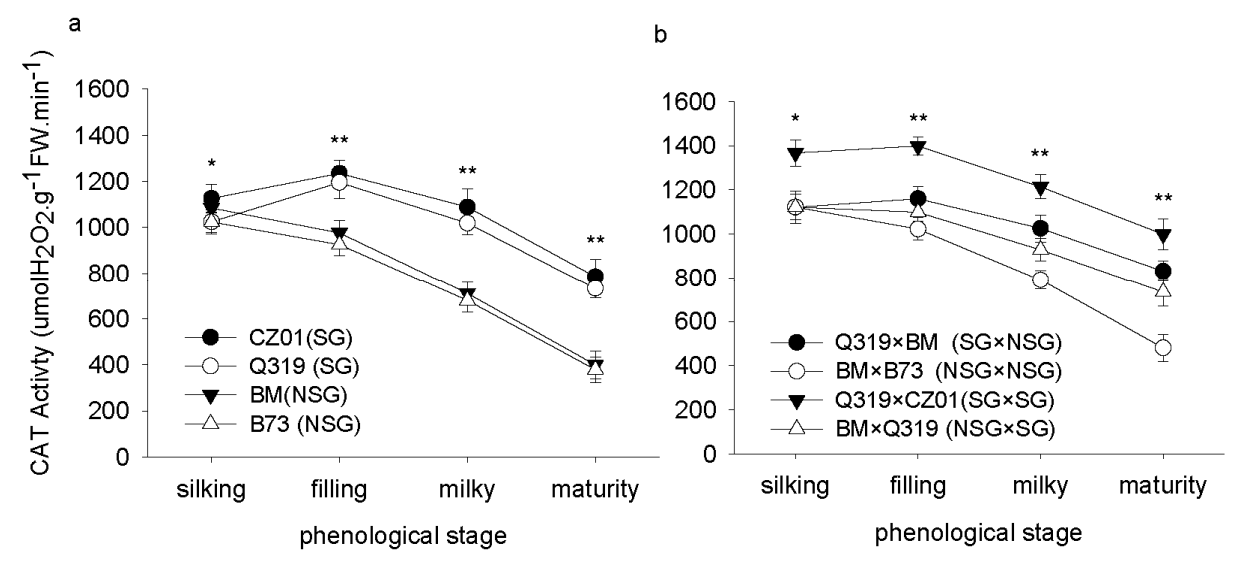

Figure 3. Catalase (CAT) activity in ear leaves from different maize genetic backgrounds after silking. Values are means \pm standard deviation $(n=3) .{ }^{*}$ indicates a significant difference between treatments at $p<0.05 .{ }^{* *}$ indicates a significant difference between treatments at $p<0.01$. (a) represents inbred lines, (b) represents hybrids. SG1 and SG2 are two types of maize for a higher stay-green ratio; CZ01, Q319, BM, B73 are the names of maize varieties.

\subsection{Malondialdehyde (MDA) Content after Silking}

The MDA content in maize functional leaves increased with maturation (Figure 4); it was lower at the silking stage, slightly higher at the filling stage, and then rose rapidly. This suggests that intense cell membrane oxidation took place after the filling stage. The SG parental lines showed lower MDA content than the NSG parental lines at all stages. In F1 crossed lines, the leaf MDA content was the highest in Q319 $\times$ CZ01, followed by $\mathrm{Q} 319 \times \mathrm{BM}, \mathrm{BM} \times \mathrm{Q} 319$, and $\mathrm{BM} \times \mathrm{B} 73$ for all growth stages. Thus, the SG $\times$ SG plants showed better ROS scavenging performance, with lower membrane lipid peroxide levels when compared to NSG $\times$ NSG plants. The average MDA content from silking to maturation in the SG lines was 32.35\% lower than in the NSG lines, demonstrating that the SG lines had less cell membrane oxidation and thus less cell membrane damage, better leaf protection, and slower function decline.
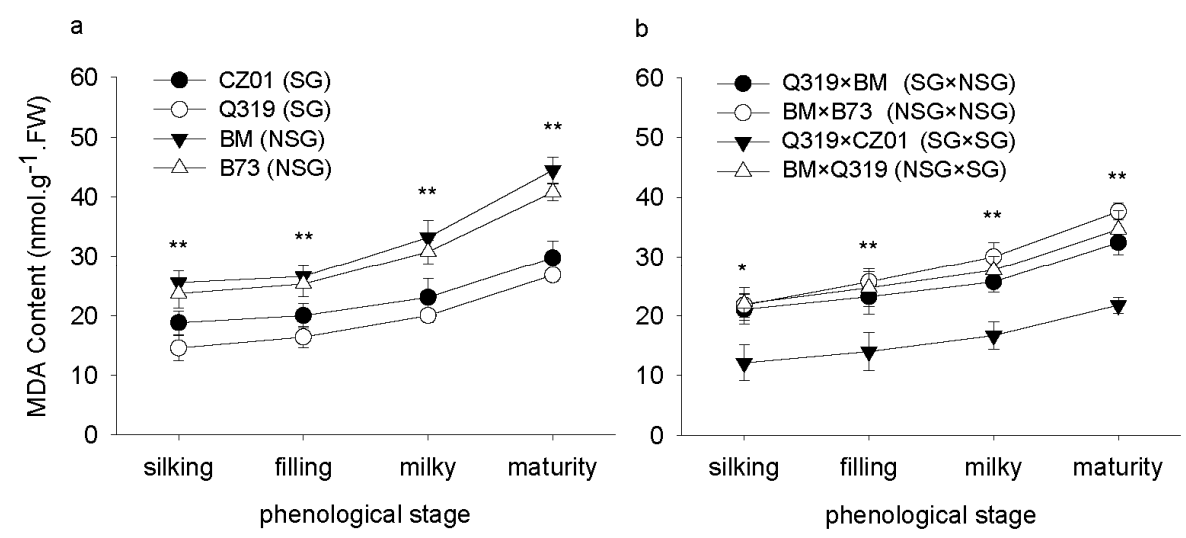

Figure 4. Malondialdehyde (MDA) content in ear leaves from different maize genetic backgrounds after silking. Values are means \pm standard deviation $(n=3) .{ }^{*}$ indicates a significant difference between treatments at $p<0.05$.** indicates a significant difference between treatments at $p<0.01$. (a) represents inbred lines, (b) represents hybrids. SG1 and SG2 are two types of maize for a higher stay-green ratio; CZ01, Q319, BM, B73 are the names of maize varieties.

\subsection{Hormone Content in Leaves after Silking}

Since hormones, and, in particular, CTKs and ABA, can control senescence in maize, we measured the leaf content of the CTK, zeatin riboside (ZR), and ABA in leaves at different developmental stages. The ZR content increased first and then declined, reaching a maximum at the filling stage (Figure 5). 
The ZR content was always higher in the SG parental lines than in the NSG parental lines at every stage tested by an average of $72.65 \%$. In the F1 crossed lines, the ZR content was the highest in SG $\times$ SG, followed by SG $\times$ NSG, NSG $\times$ SG, and NSG $\times$ NSG. Furthermore, the average ZR content from silking to maturation in the SG $\times$ SG lines was $82.03 \%$ higher than in the NSG $\times$ NSG lines.
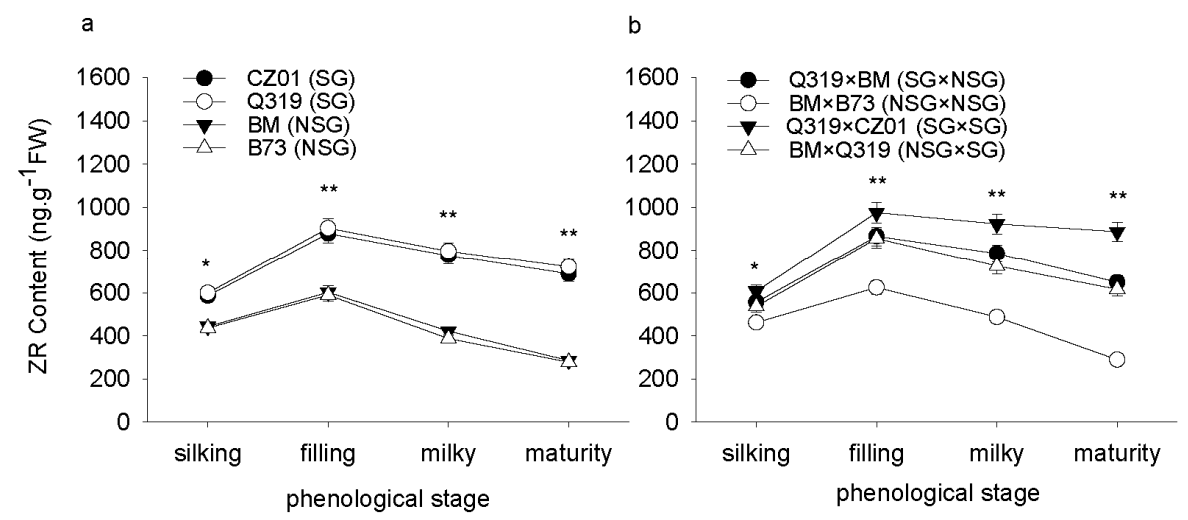

Figure 5. Zeatin riboside (ZR) content in ear leaves from different maize genetic backgrounds after silking. Values are means \pm standard deviation $(n=3) .{ }^{*}$ indicates a significant difference between treatments at $p<0.05 .{ }^{* *}$ indicates a significant difference between treatments at $p<0.01$. (a) represents inbred lines, (b) represents hybrids. SG1 and SG2 are two types of maize for a higher stay-green ratio; CZ01, Q319, BM, B73 are the names of maize varieties.

The ABA content in maize with different genetic backgrounds increased slowly from the silking to maturity stage, except for the NSG lines, where it quickly increased from the filling to the milky stage, and then went back to a slow increase (Figure 6). From the silking to the maturity period, the ABA content increased by $44.50 \%$ in the SG parental lines, compared to $51.49 \%$ in the NSG parental lines. Among the filial generations, the ABA content was the highest in the SG parental lines and SG $\times$ SG, followed by SG $\times$ NSG, NSG $\times$ SG, and NSG $\times$ NSG or NSG parental lines. Additionally, the average ABA content from silking to maturity in the SG $\times$ SG lines was $57.97 \%$ lower than in the NSG $\times$ NSG lines.
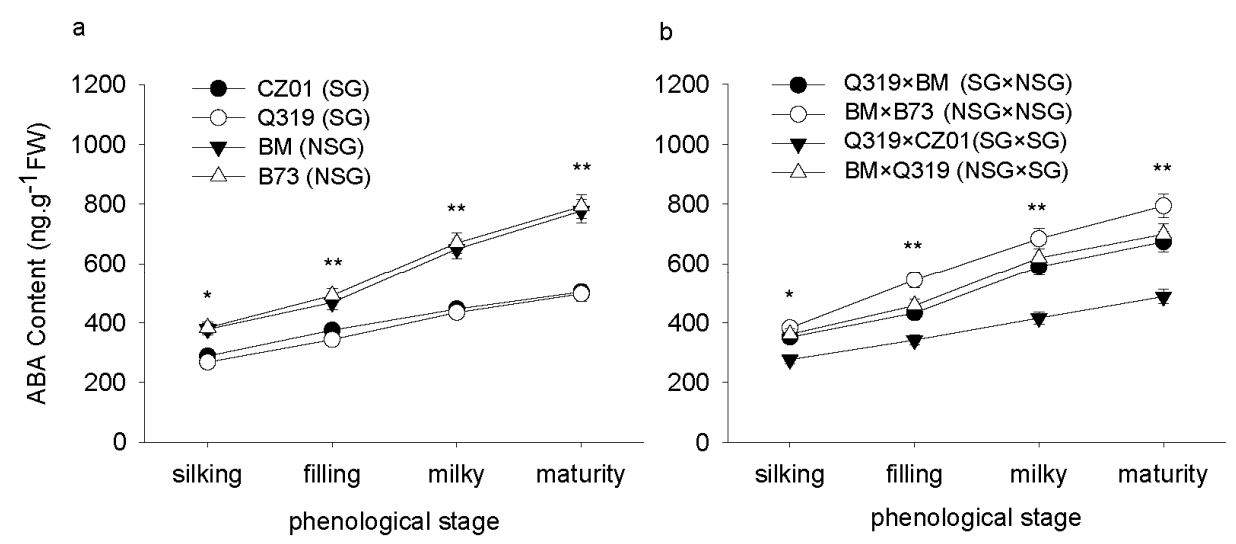

Figure 6. Abscisic acid (ABA) content in ear leaves from different maize genetic backgrounds after silking. Values are means \pm standard deviation $(n=3) .{ }^{*}$ indicates a significant difference between treatments at $p<0.05$. ${ }^{* *}$ indicates a significant difference between treatments at $p<0.01$. (a) represents inbred lines, (b) represents hybrids. SG1 and SG2 are two types of maize for a higher stay-green ratio; CZ01, Q319, BM, B73 are the names of maize varieties.

The ZR/ABA ratio was 2.5 times higher in the SG lines than that in the NSG lines (Figure 7). Moreover, the ZR to ABA ratio was the highest in $\mathrm{SG} \times \mathrm{SG}$, followed by SG $\times \mathrm{NSG}, \mathrm{NSG} \times \mathrm{SG}$, and NSG $\times$ NSG. The mean ZR/AB in SG $\times$ SG was 2.88 times higher than that of NSG $\times$ NSG. 
The increased relative ZR leaf content might be related to slower senescence and an increase in the SG period.

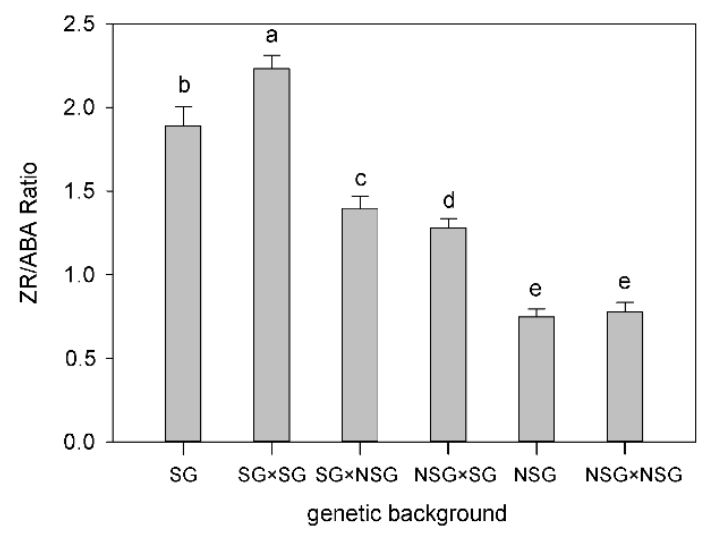

Figure 7. The ratio of zeatin riboside (ZR)/abscisic acid (ABA) in ear leaves from different maize genetic backgrounds from silking to maturity. Values are means of the four stages (silking, filling, milky, and maturity). The different letters indicate significance at the $p<0.05$ level. SG is for CZ01 and Q319, NSG is for BM and B73, SG $\times$ NSG is for Q319 $\times$ BM, NSG $\times$ NSG is for BM $\times$ B73, SG $\times$ SG is for $\mathrm{Q} 319 \times \mathrm{CZ} 1 \mathrm{1}$, and NSG $\times \mathrm{SG}$ is for $\mathrm{BM} \times \mathrm{Q} 319$.

\section{Discussion}

The development of the free radical theory by Firdovich (1975) [29], drew a lot of attention to mechanistic research into plant stress resistance and plant senescence. In this study, by measuring the activity of the antioxidant enzymes SOD, POD, and CAT, as well as by measuring the MDA content and the content of the related hormones $\mathrm{ZR}$ and $\mathrm{ABA}$, we investigated the relationship between the SG trait and the physiological delay of senescence. We also considered whether hybrid SG $\times$ NSG plants maintained this stay-green trait to some extent. We found that the activity of SOD, POD, and CAT in leaves showed a similar pattern during the later stages of maize reproduction (from silking to maturity) - a rise, followed by a decline, reaching the maximum at the filling stage. At every stage, the SG $\times$ SG hybrids displayed correspondingly higher activity than all measured enzymes compared to the NSG $\times$ NSG hybrids. Our findings were consistent with other reports in maize, wheat, and rice [30-32]. Since ROS scavenging activity is a marker of senescence, SG $\times$ SG hybrids can delay leaf senescence as a result of more active antioxidant activity through these protective enzymes, protecting leaves from yellowing. The lower MDA levels in SG $\times$ SG plants also supports our hypothesis that leaf senescence in SG $\times$ SG plants was slower due to the occurrence of less cell membrane damage. We concluded that the SG lines showed better ROS scavenging properties at all stages than the NSG lines, thus maintaining the leaves at the functional stages for extended periods. They are therefore expected to promote dry matter accumulation in the kernels.

Regarding the mechanism of leaf senescence related to ROS, we do not have direct data on the efficiency of ROS-mediated signaling in these maize plants. As ROS accompanies senescence, stay-green lines may produce lower levels of ROS, and hence, ROS-scavenging enzymes. It is possible that higher ROS scavenging activity leads to impaired ROS-mediated signaling, thus delayed senescence. The decline in SOD, POD, and CAT activity indicates that the delayed senescence in SG plants is likely to be due to the regulation of senescence at the level of ROS creation rather than on the downstream targets, for example, gene expression or protein stability. In this study, we did not address whether the SG trait is correlated with higher levels of these proteins or if this system is instead more active in these plants. Higher levels of ROS scavenging might be a side effect of higher ROS production due to the photosynthetic rate [33], rather than being the main causative effect of the SG trait. Future studies are needed to address this question and investigate the molecular mechanisms involved. 
In terms of the hormonal control of senescence, as reported earlier, ZR delays the degradation of the photosynthetic machinery, which is compatible with a greener leaf color [17]. At the same time, previous reports have also indicated that the overaccumulation of $Z \mathrm{R}$ due to the overexpression of biosynthetic genes results in plants oversensitive to abiotic stress. While our data indicate that the absolute ZR levels were always in the physiological range [34], future work will need to keep these higher concentrations of $\mathrm{ZR}$ in mind, particularly when breeding high-yield hybrids. The levels of ABA measured in our study were compatible with previous studies, indicating that this hormone acts as a senescence-promoting factor [35]. As the SG $\times$ SG plants presented at lower levels of ABA when compared to other hybrids, this likely indicates that these plants have a delayed, genetically-induced senescence. Both of these hormones are involved in the control of leaf senescence and have a direct influence on photosynthesis [16]. However, with our experimental setup, it was not possible to provide evidence as to whether the change in hormonal levels was causative of or dependent on the SG trait. We observed a decline in the ZR level in all plants, in agreement with the notion that a decrease in cytokinin levels is a signal for the initiation of senescence [36]. At the same time, ABA levels are modulated by abiotic stress [37], leaving open the possibility that the lower levels in SG plants might be related to inefficient stress responses. In our experiment, we did not test our plants under abiotic stress conditions. It might be interesting to measure whether the production of ABA is similar to NSG under stress, to better understand the physiological meaning of our data. Therefore, while it is likely that the exogenous application of these hormones before the silking stage might change the photosynthetic efficiency of the plants, these experiments would also help to improve our understanding of the roles of $\mathrm{ZR}$ and ABA in SG plants. Further investigation is therefore needed to clarify the significance of the unbalanced levels of these hormones in SG plants.

The NSG $\times$ SG and SG $\times$ NSG plants partially retained the characteristics of the SG $\times$ SG plants, with ROS scavenging activity and hormones at intermediate levels between SG $\times$ SG plants and NSG $\times$ NSG plants. Our data provided a starting point to create hybrid SG plants that preserve their photosynthetic characteristics while potentially introducing other beneficial traits through crossing. Previous studies have indicated that both dominance and additive effects, with a predominance of the latter [5], control the inheritance of the SG trait. Our data are in agreement with this notion. The ROS scavenging profile and the hormonal level of our experiments indeed seem to suggest that the SG trait is inherited in an additive fashion. This means that not only can the SG trait be crossed to obtain varieties to combine this trait with others, but also that crossing different SG varieties could result in better SG cultivars.

In summary, by comparing the activity of the SOD, POD, and CAT enzymes as well as the MDA content, we found that the SG lines show better ROS scavenging properties at all stages of development than NSG lines. We also found that the SG trait was accompanied by better ROS scavenging, higher ZR, and a lower ABA content in leaves. Moreover, our study indicated that the SG trait was at least partially retained in the SG $\times$ NSG hybrid, providing basic information for the use of the SG trait in hybrid breeding to maintain the leaf at functional stages for an extended period and is thus expected to promote dry matter accumulation in the kernels.

\section{Conclusions}

By comparing the activity of the SOD, POD, and CAT enzymes and the MDA content, we found that the SG lines showed better ROS scavenging properties at all stages than the NSG lines. We also found that the SG trait was accompanied by better ROS scavenging, a greater ZR content, and a lower ABA content in leaves. Accordingly, stay-green maize appears to eliminate active oxygen effectively, which is beneficial for slowing leaf senescence and improving dry matter accumulation, which provides a physiological basis for higher maize yields. Our research is meaningful for maize senescence regulation and good variety selection for stay-green maize.

Author Contributions: Writing-Original Draft Preparation, C.L. and Z.L.; Resources, T.L., Q.W., and Y.S.; Data Analysis, H.Z. (Hui Zhang) and H.Z. (Haijun Zhao); Writing-Editing, H.D. and K.L. 
Funding: This research was financially supported by the National Key R\&D Program of China (2018YFD0300602), the Shandong Province Innovation Team Project of Modern Agricultural Industry Technology System (SDAIT-01-021-11, SDAIT-01-021-07), and the Key Development Plan Project of Shandong Province (2016GNC111022, 2016GNC111004).

Conflicts of Interest: The authors declare no conflict of interest.

\section{References}

1. Wang, K.J.; Dong, S.T.; Hu, C.H.; Liu, K.; Zhang, J.W. The evolution of physiological characteristics of maize root during varietal replace in China, 1905s to 1990s II. Changes of the protective enzyme activities and lipid peroxidation. Acta Agron. Sin. 2002, 28, 384-388.

2. Jagadish, K.S.V.; Kavi, K.P.B.; Bahuguna, R.N.; Nicolaus, V.W.; Nese, S. Staying alive or going to die during terminal senescence-an enigma surrounding yield stability. Front. Plant Sci. 2015, 6, 1070. [CrossRef] [PubMed]

3. Antonietta, M.; Fanello, D.D.; Acciaresi, H.A.; Guiamet, J.J. Senescence and yield responses to plant density in stay green and earlier-senescing maize hybrids from Argentina. Field Crops Res. 2014, 155, 111-119. [CrossRef]

4. Gregersen, P.L.; Culetic, A.; Boschian, L.; Krupinska, K. Plant Senescence and Crop Productivity. Plant Mol. Biol. 2013, 82, 603-622. [CrossRef] [PubMed]

5. Belícuas, P.R.; Aguiar, A.M.; Bento, D.A.V.; Câmara, T.M.M.; Junior, C.L.S. Inheritance of the Stay-Green Trait in Tropical Maize. Euphytica 2014, 198, 163-173. [CrossRef]

6. Renu, K. Leaf senescence and abiotic stresses share reactive oxygen species-mediated chloroplast degradation. Protoplasma 2012, 249, 469-481.

7. Smith, K.; Craven, M.; Mclaren, N.W.; Bemer, J.M. Effects on fungicide application on sorghum yield, leaf senescence and photosynthetic efficiency in South Africa. S. Afr. J. Bot. 2015, 121, 201. [CrossRef]

8. Vietor, D.M.; Rhodes, G.A.; Rooney, W.L. Relationship of phenotypic variation in sorghum to nutritive value of crop residues. Field Crops Res. 2010, 118, 243-250. [CrossRef]

9. Spano, G.; Di, F.N.; Perrotta, C.; Platani, C.; Ronga, G.; Lawlor, D.W.; Napier, J.A.; Shewry, P.R. Physiological characterization of 'stay green' mutants in durum wheat. J. Exp. Bot. 2003, 54, 1415-1420. [CrossRef] [PubMed]

10. Ma, D.L.; Xie, R.Z.; Zhang, F.L.; Li, J.; Li, S.M.; Long, H.L.; Liu, Y.; Guo, Y.Q.; Li, S.K. Genetic contribution to maize yield gain among different locations in China. Maydice 2016, 6, 1070.

11. Zhang, X.X.; Wang, M.; Wu, T.T.; Wu, C.X.; Jiang, B.J.; Guo, C.H.; Han, T.F. Physiological and molecular studies of stay-green caused by pod removal and seed injury in soybean. Crop J. 2016, 6, 435-443. [CrossRef]

12. Wingler, A.; Von, S.A.; Leegood, R.C.; Lea, P.J.; Quick, W.P. Regulation of leaf senescence by cytokinin, sugars, and light. Effects on NADH-dependent hydroxypyruvate reductase. Plant Physiol. 1998, 116, 329-335. [CrossRef]

13. Hu, T.; Yuan, L.; Wang, J.; Kang, S.; Li, F. Antioxidation responses of maize roots and leaves to partial root-zone irrigation. Agric. Water Manag. 2010, 98, 164-171. [CrossRef]

14. Blumwald, E. Sodium transport and salt tolerance in plants. Curr. Opin. Cell Biol. 2000, 12, 431-434. [CrossRef]

15. Suzuki, N.; Shai, K.; Ron, M.; Gad, M. ROS and redox signalling in the response of plants to abiotic stress. Plant Cell Environ. 2012, 35, 259-270. [CrossRef] [PubMed]

16. He, P.; Jin, J.Y. Effect of $\mathrm{N}$ and $\mathrm{K}$ nutrition on changes of endogenous hormone and metabolism of active oxygen during leaf senescence in spring maize. J. Plant Nutr. Fertil. 1999, 4, 289-296.

17. Humbeck, K.; Quast, S.; Krupinska, K. Functional and molecular changes in the photosynthetic apparatus during senescence of flag leaves from field-grown barley plants. Plant Cell Environ. 1996, 19, 337-344. [CrossRef]

18. Markovich, O.; Steiner, E.; Kouřil, Š.; Tarkowski, P.; Aharoni, A.; Elbaum, R. Silicon promotes cytokinin biosynthesis and delays senescence in Arabidopsis and Sorghum. Plant Cell Environ. 2017, 40, 1189-1196. [CrossRef] [PubMed] 
19. Raines, T.; Shanks, C.; Cheng, C.Y.; McPherson, D.; Argueso, C.T.; Kim, H.J.; Franco-Zorrilla, J.M.; López-Vidriero, I.; Solano, R.; Vaňková, R.; et al. The cytokinin response factors modulate root and shoot growth and promote leaf senescence in Arabidopsis. Plant J. 2016, 85, 134-147. [CrossRef] [PubMed]

20. Borrell, A.K.; Hammer, G.L.; Henzell, R.G. Does maintaining green leaf area in sorghum improve yield under drought? II. Dry matter production and yield. Crop Sci. 2000, 40, 1037-1048. [CrossRef]

21. Liu, K.C.; Dong, S.T.; Zhao, H.J.; Wang, Q.C.; Li, Z.X.; Liu, X.; Zhang, H. Leaf stay-green traits in Chinese maize inbred lines and their relationship with grain yield. Acta Agron. Sin. 2009, 35, 1662-1671. [CrossRef]

22. Wang, K.J.; Hu, C.H.; Dong, S.T.; Liu, K.C.; Sun, Q.Q. Changes of the protective enzyme activities and lipid peroxidation after anthesis among maize varieties planted in different years. Acta Agron. Sin. 1999, 25, 700-706.

23. Zhang, Z.; Li, G.; Gao, H.; Zhang, L.; Yang, C.; Liu, P.; Meng, Q. Characterization of Photosynthetic Performance during Senescence in Stay-Green and Quick-Leaf-Senescence Zea mays L. Inbred Lines. PLOS ONE 2012, 7, e42936. [CrossRef] [PubMed]

24. Liu, C.; Zhao, H.; Dong, S.; Wang, Q.; Li, Z.; Liu, K. Study on Characteristics of Nitrogen Metabolism in Diallel Cross Generation of Different Maize Genotypes After Silking. Sci. Agric. Sin. 2014, 47, 33-42.

25. Winterbourn, C.; Hawkins, R.; Brian, M.; Carrell, R. The estimation of red cell superoxide dismutase activity. J. Lab. Clin. Med. 1975, 85, 337-341. [PubMed]

26. Zhang, Z.O.; Pang, X.Q.; Xue, W.D.; Ji, Z.L.; Jiang, Y.M. Role of peroxidase in anthocyanin degradation in litchi fruit pericarp. Food Chem. 2005, 90, 47-52. [CrossRef]

27. Schmedes, A.; Hølmer, G. A new thiobarbituric acid (TBA) method for determining free malondialdehyde (MDA) and hydroperoxides selectively as a measure of lipid peroxidation. J. Am. Oil Chem. Soc. 1989, 66, 813-817. [CrossRef]

28. Liu, K.C. Study on Physiological Mechanism and Inheritance of Leaf Stay-Green Traits of Different Maize (Zea mays L.) Genotypes; Shandong Agricultural University: Tai'an, China, 2003.

29. Fridovich, I. Superoxide dismutases. Annu. Rev. Biochem. 1975, 44, 147-159. [CrossRef] [PubMed]

30. Prochazkova, D.; Sairam, R.K.; Srivastava, G.C.; Singh, D.V. Oxidative stress and antioxidant activity as the basis of senescence in maize leaves. Plant Sci. 2001, 161, 765-771. [CrossRef]

31. Xue, H.; Jia, L.; Gong, Y.H.; Liu, Y.Z.; Wu, Y.H. Study on the stay-green capacity and leaf senescence of winter wheat. Acta Bot. Boreal. Occident. Sin. 2010, 30, 0336-0343.

32. Wang, F.; Liu, J.; Zhou, L.; Pan, G.; Li, Z.; Zaidi, S.H.R.; Cheng, F. Senescence-specific change in ROS scavenging enzyme activities and regulation of various SOD isozymes to ROS levels in Psf mutant rice leaves. Plant Physiol. Biochem. 2016, 109, 248-261. [CrossRef] [PubMed]

33. Asada, K. Production and scavenging of reactive oxygen species in chloroplasts and their functions. Plant Physiol. 2006, 141, 391-396. [CrossRef] [PubMed]

34. Vyroubalová, Š.; Václavíková, K.; Turečková, V.; Novák, O.; Šmehilová, M.; Hluska, T.; Ohnoutková, L.; Frébort, I.; Galuszka, P. Characterization of new maize genes putatively involved in cytokinin metabolism and their expression during osmotic stress in relation to cytokinin levels. Plant Physiol. 2009, 151, $433-447$. [CrossRef] [PubMed]

35. Badenoeh-Jones, J.; Parker, C.W.; Letham, D.S. Effect of cytokinin supplied via the xylem at multiples of endogenous concentration on transpiration and senescence in derooted seedlings of oat and wheat. Plant Cell Environ. 1996, 19, 504-516. [CrossRef]

36. Gan, S.; Amasino, R.M. Inhibition of Leaf Senescence by Autoregulated Production of Cytokinin. Science 1995, 270, 1986-1988. [CrossRef] [PubMed]

37. Xiong, L.; Zhu, J.K. Regulation of abscisic acid biosynthesis. Plant Physiol. 2003, 133, 29-36. [CrossRef] [PubMed]

(C) 2018 by the authors. Licensee MDPI, Basel, Switzerland. This article is an open access article distributed under the terms and conditions of the Creative Commons Attribution (CC BY) license (http:/ / creativecommons.org/licenses/by/4.0/). 